\title{
Separation of transition metals in nonaqueous media with capillary electrophoresis
}

\author{
Feng $\mathrm{Qu}^{\mathrm{a}} \mathrm{b}$, Jin-Ming $\operatorname{Lin}^{\mathrm{a}, *}$ \\ ${ }^{a}$ Research Center for Eco-Environmental Sciences, Chinese Academy of Sciences, P.O. Box 2871, Beijing 100085, China \\ ${ }^{\mathrm{b}}$ School of Life Science \& Technology, Beijing Institute of Technology, P.O. Box 327, Beijing 100081, China
}

Available online 18 November 2004

\begin{abstract}
The separation of transition metal $\mathrm{Ni}^{2+}, \mathrm{Cu}^{2+}, \mathrm{Co}^{2+}, \mathrm{Zn}^{2+}, \mathrm{Cd}^{2+}$ and $\mathrm{Fe}^{3+}$ in methanol was investigated by using different types of organic acids as complexing agents. In pure methanol, the weaker and simpler acetic, propionic, butyric and valeric acids could enhance metal ions selectivity by increasing acid concentration and metal ions could be separated with high efficiency. However, hydroxycarboxylic acids obviously made separation efficiency worse. The effect of mixed organic acids, mixture solvent (methanol-acetonitrile, methanol-water) on metal ions separation was discussed further. The advantages of using nonaqueous solvent over aqueous for metal ions separation were shown finally. (c) 2004 Elsevier B.V. All rights reserved.
\end{abstract}

Keywords: Nonaqueous capillary electrophoresis; Transition metals; Organic acids

\section{Introduction}

Capillary electrophoresis (CE) has been developing very rapidly since the 1990s. The number of papers dealing with analysis of metal ions has increased quickly. The traditional approach to the separation of metal ions, ion chromatography (IC), is partially being replaced by CE due to its speed, resolving power, minimal sample and reagent requirements and adaptability to a variety of applications using different separation conditions. The greater simplicity in operation of $\mathrm{CE}$ is another advantage. For example, IC requires post-column chelating reagent mixing system for transition metal ions [1], however, the simultaneous separation of routine transition metals, even lanthanide metals with simple indirect detection can be easily achieved with CE [2-9]. The separation process is easily carried out through on-capillary complexation by using weak complexing agents of organic acids.

However, unlike in aqueous, the effect of organic acids in pure organic solvent should be different since the ionization constants of organic acids change in nonaqueous media. Besides that, electrophoretic mobilities of metal ions are also

\footnotetext{
* Corresponding author. Tel.: +86 1062841953 ; fax: +86 1062841953 .

E-mail address: jmlin@mail.rcees.ac.cn (J.-M. Lin).
}

affected due to the interaction between metal ions with polar solvent molecule through ion-dipole bonds. The existence of solvation shell, which is formed around the center ions alters actual size and effective charge of ions, therefore, changes their mobilities. In principle, the solvent solvates all kinds of ions, some to a greater extent than others, depending on the specific properties of the central ion regarding a certain solvent.

Methanol, a protic solvent, is often used in electrolyte to improve the separation selectivity. The favorite amount added is between $5 \%$ and $30 \%$, and higher percentage of methanol can cause electrical breakdown [10,11]. However, pure methanol not only changes the acid dissociation constant by many orders of magnitude compared with that in water but also offers potential for adjustment of relative migration rates via change in solvation-ion interaction. So far, only a few articles report the separation of metal ions in pure methanol solvent with acetic acid as complexing agent $[2,12,13]$. In this paper, nine different types of organic acids (chain acids, hydroxycarboxylic acids and benzoic acid) are investigated for the separation of transition metal $\mathrm{Fe}^{3+}, \mathrm{Ni}^{2+}$, $\mathrm{Cu}^{2+}, \mathrm{Co}^{2+}, \mathrm{Zn}^{2+}$ and $\mathrm{Cd}^{2+}$. The influence of mixture solvent (methanol-acetonitrile, methanol-water) on metal ions migration behavior is discussed. The advantages of using non- 
aqueous solvent over aqueous for metal ions separation are shown.

\section{Experimental}

\subsection{Instrumentation}

Experiments were performed with a Beckman P/ACE MDQ system (USA), which comprises a CE unit with diode array detector and workstation. Separations were carried out on fused-silica capillaries of $57 \mathrm{~cm}(50 \mathrm{~cm}$ effective length) $\times 100 \mu \mathrm{m}$ i.d. (Yongnian Optical Fiber Factory, Heibei, China).

\subsection{Chemicals}

All reagents were of analytical grade. Metal ion standard stock solutions were prepared by dissolving metal salts $\mathrm{CuSO}_{4} \cdot 5 \mathrm{H}_{2} \mathrm{O}, \mathrm{NiCl}_{2} \cdot 6 \mathrm{H}_{2} \mathrm{O}, \mathrm{CoCl}_{2} \cdot 6 \mathrm{H}_{2} \mathrm{O}, \mathrm{ZnCl}_{2}$, $\mathrm{CdCl}_{2} \cdot \mathrm{H}_{2} \mathrm{O}, \mathrm{FeCl}_{3} \cdot 6 \mathrm{H}_{2} \mathrm{O}$ in methanol of a concentration of $500 \mu \mathrm{g} / \mathrm{ml}$. Nine organic acids: formic, acetic, propionic, butyric, valeric acid, glycolic, lactic, 2-hydroxyisobutyric acid (HIBA), benzoic acid, and imidazole were from various vendors: Beijing Chemical Company (Beijing, China), Acros Organics and Kanto Chemicals (Tokyo, Japan). Methanol and acetonitrile were of chromatographic grade, purchased from Siyou Biology Medical Co. (Tianjin, China).

\subsection{Electrophoretic procedures}

The new capillary was rinsed with methanol, water, $1 \mathrm{M}$ $\mathrm{HCl}$ and water for $5 \mathrm{~min}$, and then activated with $1 \mathrm{M} \mathrm{NaOH}$ and water for $30 \mathrm{~min}$, respectively. Between injections the capillary was rinsed with carrier electrolyte for $3 \mathrm{~min}$. The separation was run at a constant temperature of $25^{\circ} \mathrm{C}$ and the voltage applied was $20 \mathrm{kV}$. The detection wavelength utilized was $191-250 \mathrm{~nm}$. Pressure injection was performed using $0.5 \mathrm{psi}$ for $5 \mathrm{~s}(1 \mathrm{psi}=6894.76 \mathrm{~Pa})$.

\section{Results and discussion}

\subsection{Influence of organic acids type}

Monoprotic organic acids (HA), chain carboxylic acids (formic, acetic, propionic, butyric and valeric acid), hydroxycarboxylic acids (glycolic, lactic, HIBA) and aromatic acid (benzoic acid) were selected as complexing agents. At same acids concentration, ligand $\mathrm{A}^{-}$concentration was determined by acids dissociation constants $\mathrm{p} K_{\mathrm{a}}$ which related to the type of acids (see Table 1). With acids concentration increase, metal ions apparent mobilities changed due to the combined influence of electroosmotic flow (EOF) and complexation. So, the electrophoretic mobilities of metal ions exhibited the complexation degree between metal ions and ligand $\mathrm{A}^{-}$after eliminating the variant of solution EOF due to acids concentration increase.
Table 1

Organic acid dissociation constants in water

\begin{tabular}{ll}
\hline Acid & $\mathrm{p} K_{\mathrm{a}}$ \\
\hline Formic acid & 3.751 \\
Acetic acid & 4.756 \\
Propionic acid & 4.874 \\
Butyric acid & 4.871 \\
Valeric acid & 4.842 \\
Glycolic acid & 3.831 \\
Lactic acid & 3.858 \\
HIBA & 3.991 \\
Benzoic acid & 4.170 \\
\hline
\end{tabular}

\subsubsection{Chain carboxylic acid and benzoic acid}

In order to testify the effect of carbon chain length on complexation between acids and metal ions, acetic, propionic, butyric and valeric acid were used as complexing agents. According to their $\mathrm{p} K_{\mathrm{a}}$ values in water, it could be estimated that their dissociation constants in methanol are very close, so the dissociation situations of their carboxyl which determined the complexing reaction were similar. Our experiments demonstrated that the complexation had no relations to the carbon chain length, the mobilities of metal ions were well in agreement at same acid concentration with above four acids as complexing agents. Fig. 1 shows the influence of propionic acid on mobilities. Mobilities of divalent ions $\mathrm{Ni}^{2+}, \mathrm{Cu}^{2+}, \mathrm{Co}^{2+}, \mathrm{Zn}^{2+}$ and $\mathrm{Cd}^{2+}$ followed the order $\mathrm{Ni}^{2+}>\mathrm{Cu}^{2+}>\mathrm{Co}^{2+}>\mathrm{Zn}^{2+}>\mathrm{Cd}^{2+}$. With acids concentration increase, their mobilities decreased obviously. The steepness of the decline depended on the magnitude of the formation constants [11]. The reciprocal of their mobilities against the concentration showed straight lines, whose slopes were related to complexing formation constants. From the slopes value, it could be concluded that their formation constants were in the reverse order.

Unlike divalent ions, the mobility of $\mathrm{Fe}^{3+}$ exhibited no apparent decrease when acid concentration was increased, which means that nearly no complexation occurred between $\mathrm{Fe}^{3+}$ and acids. Considering the charge/size ratio, $\mathrm{Fe}^{3+}$ should migrate faster than divalent ions. However, $\mathrm{Fe}^{3+}$ had the mobility smaller than $\mathrm{Ni}^{2+}$ and $\mathrm{Cu}^{2+}$ at low acid concentration $(0.05 \mathrm{M})$. This phenomena demonstrated that the solvation effect changed its charge/size ratio significantly, mobility of $\mathrm{Fe}^{3+}$ was dramatically controlled by the solvation of methanol. With propionic acid concentration increasing

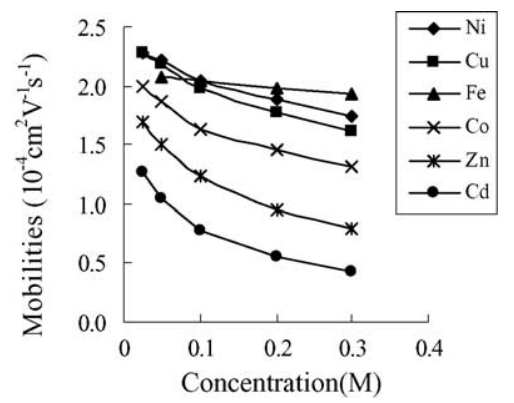

Fig. 1. Dependence of mobilities on propionic acid concentration. 
from $0.05 \mathrm{M}$ to $0.3 \mathrm{M}$, the resolution between $\mathrm{Ni}^{2+}, \mathrm{Cu}^{2+}$ and $\mathrm{Fe}^{3+}$ was improved at $0.15 \mathrm{M}$, since the mobilities of $\mathrm{Ni}^{2+}$ and $\mathrm{Cu}^{2+}$ decreased rapidly due to complexing with ligand $\mathrm{A}^{-}$. In Fig. 2, electropherogram (a) shows the separation of $\mathrm{Ni}^{2+}, \mathrm{Cu}^{2+}, \mathrm{Co}^{2+}, \mathrm{Zn}^{2+}, \mathrm{Cd}^{2+}$ and $\mathrm{Fe}^{3+}$ with propionic acid as complexing agent.

Formic acid, the strongest acid giving the smallest $\mathrm{p} K_{\mathrm{a}}$, made the mobilities of metal ions decrease sharply in a narrow concentration range $0.01-0.025 \mathrm{M}$. When the concentration reached $0.05 \mathrm{M}$, only $\mathrm{Cu}^{2+}, \mathrm{Zn}^{2+}$ and $\mathrm{Ni}^{2+}$ were detected. The dramatic decrease of mobilities at low concentration indicated the strong complexing interaction between formic acid and metal ions.

Benzoic acid, the aromatic acid with no substituent, was considered as a comparable acid of formic acid and acetic acid. Since the $\mathrm{p} K_{\mathrm{a}}$ of benzoic acid was between that of formic and acetic acid, benzoic acid reacting with metal ions formed the complexes with mobilities between formic and acetic acid. Unlike other organic acids, benzoic acid has UV absorbance itself, which overlaid imidazole's and made the maximum detection wavelength for $\mathrm{Ni}^{2+}, \mathrm{Zn}^{2+}$ and $\mathrm{Cd}^{2+}$ shift from $191 \mathrm{~nm}$ to $205 \mathrm{~nm}$. But for $\mathrm{Cu}^{2+}, \mathrm{Fe}^{3+}$ and $\mathrm{Co}^{2+}$, the biggest peaks were showed at $250 \mathrm{~nm}$. The use of benzoic acid caused lower sensitivity than other acids.

\subsubsection{Hydroxycarboxylic acid}

Comparing the pairs, glycolic and acetic acid, lactic and propionic acid, HIBA and butyric acid, hydroxyl not only increased the acid dissociation and lowered the $\mathrm{p} K_{\mathrm{a}}$ but also provided another binding group for metal ions. Both hydroxyl and carboxyl provided coordination sites for metal ion. Their conjugated interaction made the complexation much stronger than acids with only one carboxyl.

The complexation between ions and hydroxycarboxylic acids was apparently different from chain carboxylic acids. Comparing the mobilities of metal ions by using $0.005 \mathrm{M}$ HIBA with $0.3 \mathrm{M}$ propionic acid, the mobilities of $\mathrm{Ni}^{2+}$ and $\mathrm{Co}^{2+}$ were much smaller, $\mathrm{Cu}^{2+}$ and $\mathrm{Zn}^{2+}$ gave comparable results and $\mathrm{Cd}^{2+}$ was much larger. Therefore, a small amount of hydroxycarboxylic acids caused ions mobilities change significantly. In Fig. 2, electropherogram (b) shows the separation with $0.005 \mathrm{M}$ HIBA as complexing agent. Comparing with electropherogram (a), metal ions migration order changed to $\mathrm{Cu}^{2+}, \mathrm{Cd}^{2+}, \mathrm{Zn}^{2+}, \mathrm{Ni}^{2+}, \mathrm{Co}^{2+}$ and $\mathrm{Fe}^{3+}$ could not be detected.

Using hydroxycarboxylic acids as complexing agents, the separation efficiencies of metal ions were worse than that of chain carboxylic acids, tailing peaks were observed. Since the electrophoretic mobilities of metal ions are determined by the distribution among various possible complexed forms, poor peak shape might be attributed to slow complexation equilibria between different complexed species with hydroxycarboxylic acids.

\subsubsection{Mixed complexing agents}

Adding small amount of HIBA $(0.001-0.005 \mathrm{M})$ in $0.15 \mathrm{M}$ acetic acid electrolyte solution, where acetic acid acted as the main complexing agent and HIBA as the auxiliary one, the migration order remained until HIBA concentration was higher than $0.004 \mathrm{M}$. When HIBA reached $0.005 \mathrm{M}$, the migration order changed to $\mathrm{Cu}^{2+}, \mathrm{Ni}^{2+}, \mathrm{Zn}^{2+}, \mathrm{Co}^{2+}$ and $\mathrm{Cd}^{2+}$ (Fig. 3). The apparent mobilities decline of $\mathrm{Ni}^{2+}, \mathrm{Cu}^{2+}$ and

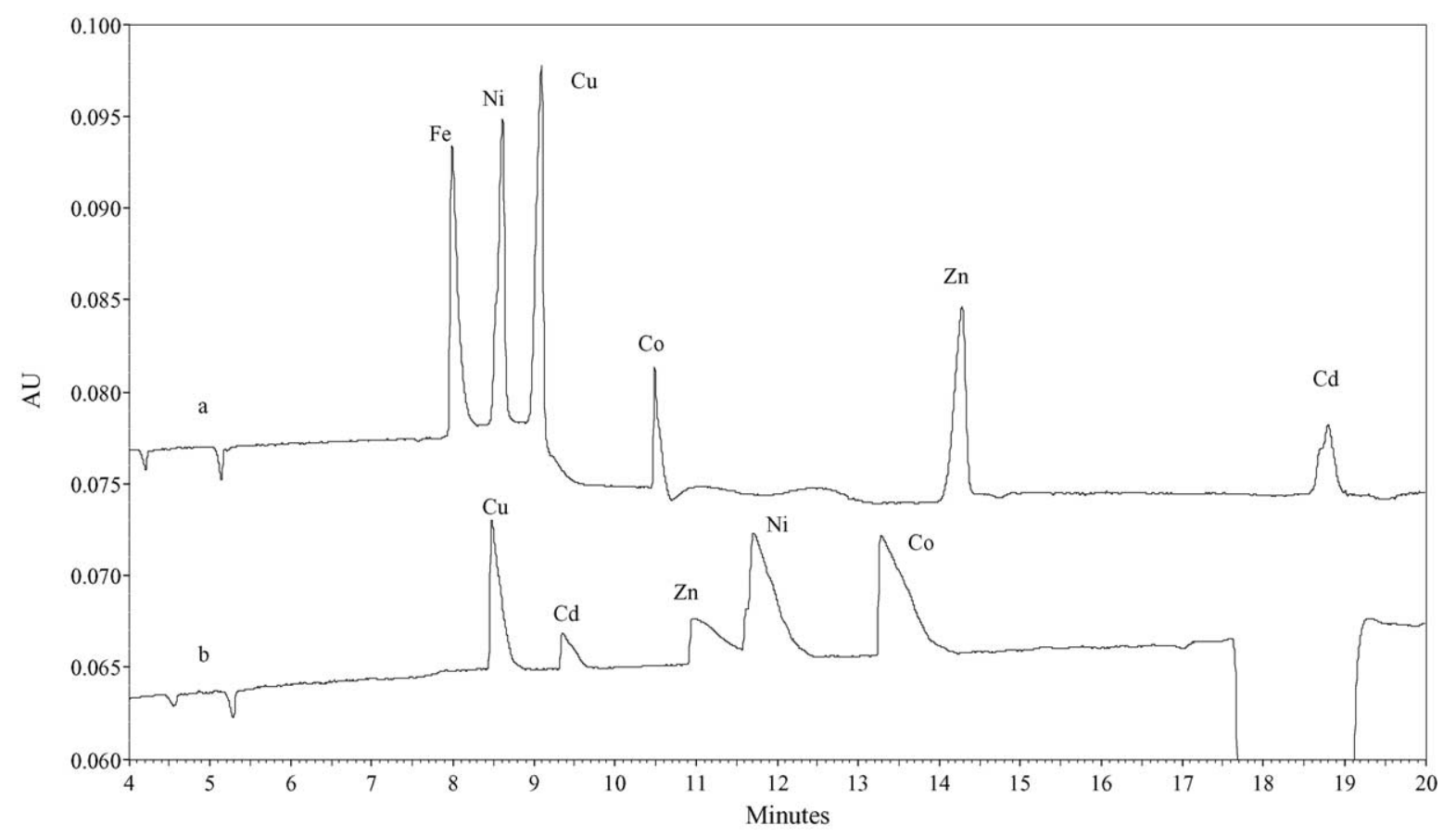

Fig. 2. Electropherograms of metal ions separation. Electrolyte: $\mathrm{a}=0.3 \mathrm{M}$ propionic acid with $0.01 \mathrm{M}$ imidazole; $\mathrm{b}=0.005 \mathrm{M}$ HIBA with $0.01 \mathrm{M}$ imidazole, $191 \mathrm{~nm}, 20 \mathrm{kV}$. 


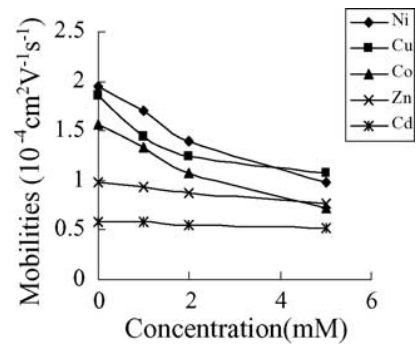

Fig. 3. Competitive complexation of HIBA and acetic acid with metal ions.

$\mathrm{Co}^{2+}$ showed the competitive complexation of HIBA with acetic acid and the coordinate effect of hydroxyl. In contrast to that, $\mathrm{Zn}^{2+}$ mobility decreased slightly and $\mathrm{Cd}^{2+}$ showed no difference. Fig. 4 shows the change of separation with HIBA concentration increase (a-d). Without HIBA, metal ions were well separated with good peak shape. However, with HIBA increase, separation efficiencies of $\mathrm{Ni}^{2+}, \mathrm{Cu}^{2+}$ and $\mathrm{Co}^{2+}$ were damaged obviously but $\mathrm{Zn}^{2+}$ and $\mathrm{Cd}^{2+}$ were kept relatively. Above results illustrated that carboxyl was the main binding group for $\mathrm{Zn}^{2+}$ and $\mathrm{Cd}^{2+}$, so the complexed species with acetic acid were stable. In contrast to that, the conjugation interaction of hydroxyl and carboxyl played more important role for $\mathrm{Ni}^{2+}, \mathrm{Cu}^{2+}$ and $\mathrm{Co}^{2+}$, the adding of HIBA resulted in the competitive complexation with acetic acid. Their poor peak shape was attributed to the possible complexed species with acetic acid and HIBA.

For all metal ions, the maximum detection wavelength with carboxylic acids as complexing agents (except benzoic acid) was $191 \mathrm{~nm}$, the sensitivity decreased with detection wavelength increase from $191 \mathrm{~nm}$ to $214 \mathrm{~nm}$.
In pure methanol, the weaker and simpler chain carboxylic acids (like acetic and propionic acid) can enhance metal ions selectivity by changing acid concentration. For the discussion of mixture solvent effect, following experiments were performed using acetic acid as complexing agent.

\subsection{Mixture solvent effect}

\subsubsection{Methanol-acetonitrile}

According to Eq. (1), the mobility of EOF is determined by the zeta potential $(\zeta)$ near the surface of the capillary, the viscosity $(\eta)$ and dielectric constant $\left(\varepsilon_{0} \varepsilon\right)$ of the background electrolyte solution.

$\mu_{\mathrm{eo}}=\frac{\varepsilon_{\mathrm{o}} \varepsilon \zeta}{\eta}$

Acetonitrile, an aproton solvent, has no influence on -SiOH dissociation of capillary wall and zeta potential near capillary wall. Since it has comparable dielectric constant (37.5) to methanol (32.7), so viscosity difference has greater effect on EOF. Acetonitrile has lower viscosity $(0.341 \mathrm{mPs}$, $\left.25^{\circ} \mathrm{C}\right)$ than methanol $\left(0.545 \mathrm{mPs}, 25^{\circ} \mathrm{C}\right)$, its addition in methanol caused the obvious increase of EOF. Fig. 5 shows the change of EOF and mobilities of metal ions with acetonitrile increase till $50 \%$. The addition of acetonitrile caused viscosity to decrease rapidly, so EOF increased sharply. Adding $10-30 \%$ acetonitrile could speed up the separation process 1.8-2.6 times. However, more acetonitrile would destroy the good resolution of metal ions. The mobilities change of metal ions $\left(\mathrm{Ni}^{2+}, \mathrm{Cu}^{2+}, \mathrm{Zn}^{2+}\right.$ showing a little increase; $\mathrm{Co}^{2+}, \mathrm{Cd}^{2+}$ showing a little decrease) might be attributed to the difference

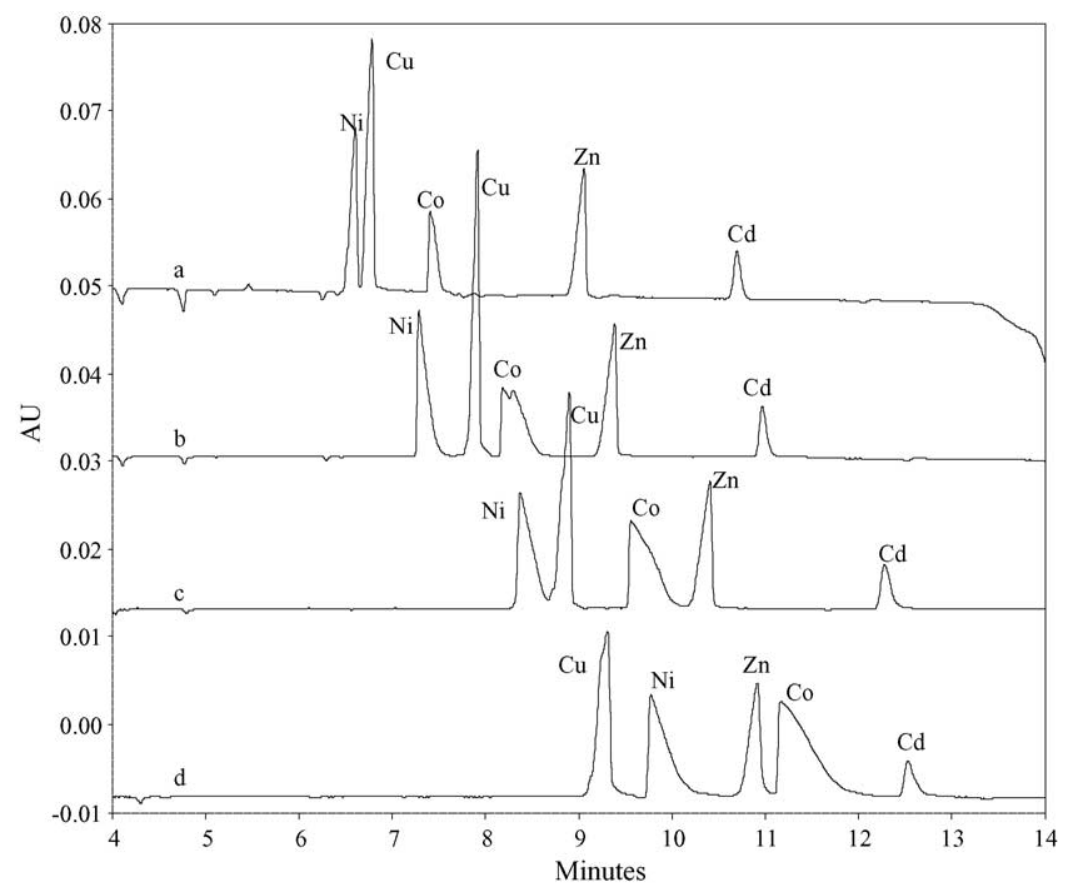

Fig. 4. Electropherograms of using mixed complexing agents. $\mathrm{a}=0.15 \mathrm{M}$ acetic acid with $0.01 \mathrm{M}$ imidazole; $\mathrm{b}=\mathrm{a}+0.001 \mathrm{M}$ HIBA; $\mathrm{c}=\mathrm{a}+0.002 \mathrm{M}$ HIBA; $\mathrm{d}=\mathrm{a}+0.005$ M HIBA. 


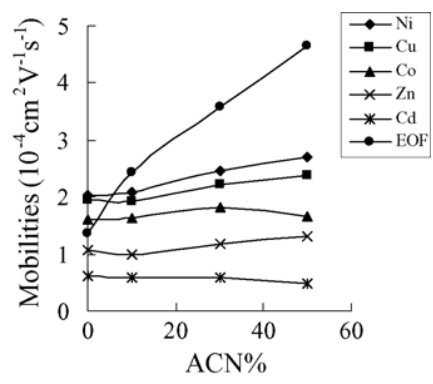

Fig. 5. Dependence of metal ion mobilities and EOF on acetonitrile.

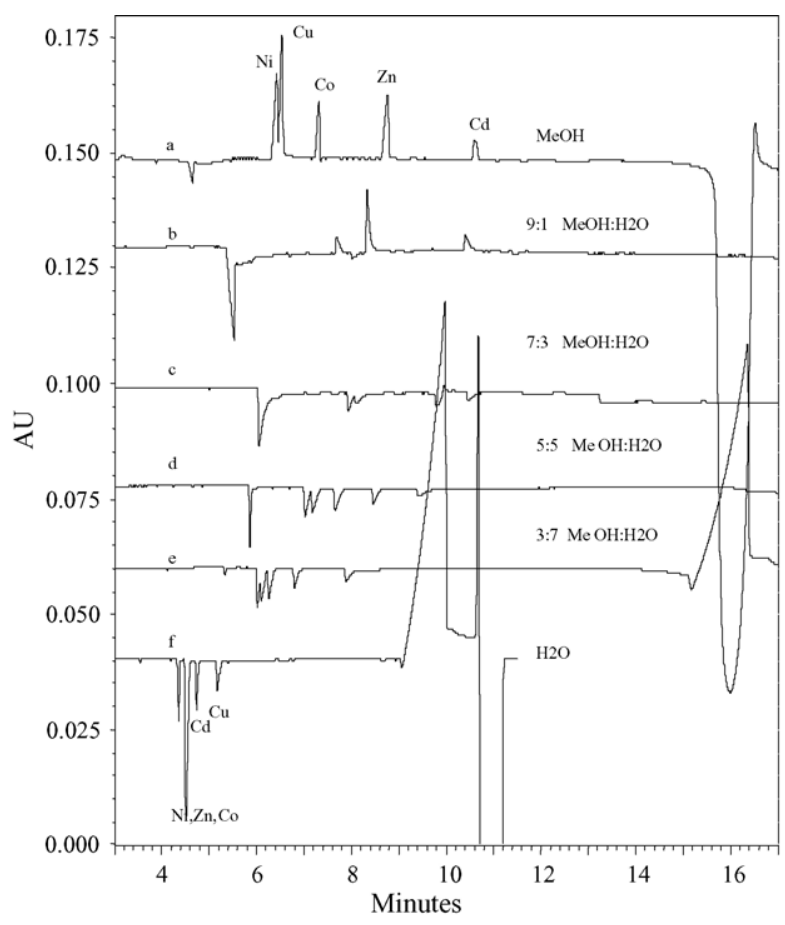

Fig. 6. Comparison of metal ions separation with pure and mixture solvent. $0.09 \mathrm{M}$ acetic acid with $0.01 \mathrm{M}$ imidazole as electrolytes.

of solvation interaction of acetonitrile to metal ions, which caused the slight difference of ions effective charge.

\subsubsection{Methanol-water}

Water, a strong proton solvent, has dramatic effect on the dissociation of $-\mathrm{SiOH}$ and zeta potential. Also, it has higher viscosity $\left(0.89 \mathrm{mPs}, 25^{\circ} \mathrm{C}\right)$ and dielectric constant (78.4) than methanol. The influence on EOF caused by adding water in methanol is determined by the combination of above affecting factors: zeta potential, dielectric constant and viscosity of the mixture solvent.

When adding $10 \%$ water in methanol, the EOF decreased $15 \%$ comparing to pure methanol. Adding $10-70 \%$ water did not cause remarked EOF change further. When water amount was higher than 70\%, EOF increased rapidly. With the same electrolyte, EOF in pure water reached the highest.

Fig. 6 shows the electropherograms with water amount increase in electrolyte. With addition of water, the separation efficiency was apparently worse (see b-e). Also, the detection of metal ions became difficult and some ions could not be detected directly. In pure water, $\mathrm{Ni}^{2+}, \mathrm{Zn}^{2+}$ and $\mathrm{Co}^{2+}$ could not be separated, however, their separation was easily obtained in pure methanol. Comparing (a) and (f), metal ions separation efficiency and detection sensitivity with pure methanol solvent were apparently better than water medium, all metal ions gave positive peaks and could be detected directly.

\subsection{Comparison of using aqueous and nonaqueous solvent}

The separation of transition metal ions in nonaqueous solvent showed the following characteristics.

(1) As we known, hydroxycarboxylic acids were most frequently used weak complexing agents for metals ion separations in aqueous solvent. However in methanol medium, simpler and weaker organic acids without $-\mathrm{OH}$ substitute group like acetic, propionic, butyric and valeric acids could be used as complexing agents and made the metal ions well separated with high efficiency. On the contrary, hydroxycarboxylic acids were not suitable at all as complexing agents.

(2) $\mathrm{Ni}^{2+}, \mathrm{Cu}^{2+}, \mathrm{Co}^{2+}, \mathrm{Zn}^{2+}$ and $\mathrm{Cd}^{2+}$ could not be well separated in aqueous, but they could be separated with high efficiency and better sensitivity in methanol. Moreover, adding acetonitrile could speed up their separation.

(3) The acid dissociation in methanol is not so susceptible as in aqueous since the dissociation constant of acid in methanol is smaller by many orders of magnitude than in water. So, the problems associated with adjusting $\mathrm{pH}$ and $\mathrm{pH}$ error between experiments could be avoided. Moreover, the electrolyte was stable and easy to prepare without need to adjust $\mathrm{pH}$.

(4) In nonaqueous solvent, the current produced was much lower, only one third of that in water, so using wider internal diameter $(100-150 \mu \mathrm{m})$ capillary were allowed and better detection sensitivity could be reached. Meanwhile, higher potential could be applied and faster separation could be achieved.

\section{Acknowledgements}

The authors wish to thank for the financial support of the National Natural Science Foundation of China (No. 20275043, No. 20377046, No. 50273046), and the National Science Fund for Distinguished Young Scholars of China (No. 20125514).

\section{References}

[1] H. Small, Ion Chromatograph, Plenum Press, New York, 1989.

[2] F. Qu, J.-M. Lin, Z. Chen, J. Chromatogr. A 1022 (2004) 217.

[3] Q. Yang, M. Jimidar, T.P. Hamoir, J. Smeyers-Verbeke, D.L. Massart, J. Chromatogr. A 673 (1994) 275. 
[4] T.L. Lin, Y.H. Lee, Y.C. Chen, J. Chromatogr. A 654 (1993) 167.

[5] A. Weston, P.R. Brown, J. Chromatogr. A 593 (1992) 289.

[6] Y. Shi, J.S. Fritz, J. Chromatogr. A 640 (1993) 473.

[7] C. Vogt, S. Conradi, Anal. Chim. Acta 294 (1994) 145.

[8] F. Foret, S. Fanali, A. Nardi, P. Bocek, Electrophoresis 11 (1990) 780.

[9] S. Conradi, C. Vogt, H. Wittrisch, G. Knobloch, G. Werner, J. Chromatogr. A 745 (1996) 103.
[10] Q. Yang, J.S. Verbeke, W. Wu, M.S. Khots, D.L. Massart, J. Chromatogr. A 688 (1994) 339.

[11] Q. Yang, Y. Zhuang, J.S. Verbeke, D.L. Massart, J. Chromatogr. A 706 (1995) 503.

[12] H.S. Moosavi, R.M. Cassidy, J. Chromatogr. A 749 (1996) 279.

[13] K.D. Altria, M. Wallberg, D. Westerlund, J. Chromatogr. B 714 (1998) 99. 